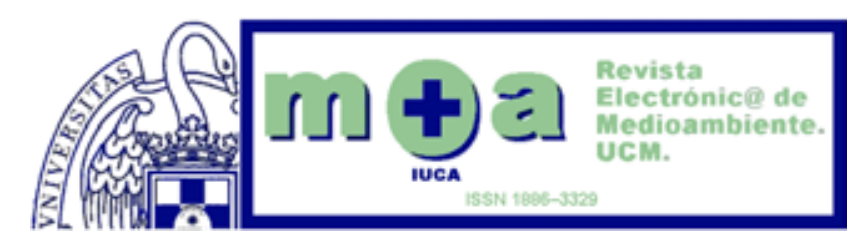

\title{
CARACTERIZACIÓN CLIMÁTICA DE LA REGIÓN DE NILI FOSSAE, MARTE
}

\section{A. Molina}

Estudiante de Doctorado. Departamento de Planetología y Habitabilidad. Centro de Astrobiología (CSIC/INTA) a.molina@csic.es

\section{M.A. de Pablo}

Profesor Ayudante Doctor. Departamento de Geología. (Universidad de Alcalá) miguelangel.depablo@uah.es

\section{Ramos}

Profesor titular. Departamento de Física. (Universidad de Alcalá) miguel.ramos@uah.es

\section{RESUMEN}

Teniendo en cuenta que conocer las condiciones climáticas de los lugares en los que amartizarán las futuras misiones robóticas a Marte es de gran importancia para el propio desarrollo de las misiones. En este trabajo analizamos datos atmosféricos extraídos de la Mars Climate Database (MCD). El objetivo es ver si éstos nos permiten conocer, con suficiente detalle, cómo es la atmósfera y el clima en una zona determinada del planeta Marte (temperaturas superficial y atmosférica, presión atmosférica o flujo de radiación, entre otras), y su variabilidad diaria y anual. Este estudio, lo aplicamos a la región de Nili Fossae, una zona de latitud, longitud y condiciones orográficas intermedias en el planeta.

Palabras clave: Clima, Atmósfera, Marte.

\section{CLIMATIC CHARACTERIZATION OF NILI FOSSAE REGION, MARS}

\begin{abstract}
Since to know the climatic conditions of landing sites for next Mars' mission it is very important for its development, we analyze here the atmospheric data from Mars Climate Database (MCD). The aim is check out if this data allow us to know how it is the atmosphere and climate of a specific area detailed enough (surficial and atmospherical temperatures, atmospheric pressures and radiation fluxes, among others), and their year and day variability. We applied this study to Nili Fossae, an area of intermediate latitude, longitude and topographic location.
\end{abstract}

Keywords: Climate, Atmosphere, Mars. 


\section{RÉSUMÉ}

Connaissant les conditions climatiques des sites d'atterrissage de Mars prochaine mission, il est très important pour son développement, sur ce travail, nous analysons les données atmosphériques de Mars Climate Database (MCD). L'objectif est de vérifier si ces données nous permettent de connaître l'atmosphère et le climat d'une zone spécifique suffisamment détaillées (températures de surface et atmosphériques, la pression atmosphérique et des flux de rayonnement, entre autres), et de sa variabilité journalière et année. Nous avons appliqué cette étude à Nili Fossae, une zone située à la latitude, la longitude et la topographie moyenne.

Mots-clés: Le climat, L'atmosphère, Mars.

\section{INTRODUCCIÓN}

El planeta Tierra, visto desde el espacio, muestra una atmosfera más que evidente, compuesta por densas capas de gases estratificados que conforman un complejo sistema generador de nubes, precipitaciones y sistemas de corrientes. Sin embargo, aunque menos conocidas, otros planetas del Sistema Solar también presentan interesantes atmosferas. Si bien en algunos casos, estas atmósferas tienen gran densidad (como en los gigantes gaseosos, Júpiter o Saturno, o en Venus), en otros casos son más livianas. Marte, el planeta más cercano a la Tierra, presenta una atmósfera menos densa que el análogo terrestre. Tiene ciertas condiciones que presentan multitud de similitudes con las de la Tierra, lo que lo hace especialmente interesante en cuanto al estudio de lo que pudo ser nuestro planeta o a lo que puede llegar a evolucionar. Por tanto el conocimiento y más profundo entendimiento de su atmósfera puede ayudarnos a conocer el funcionamiento de la atmosfera terrestre, tanto en el momento actual, como en el pasado y el futuro.

Por otro lado, los datos atmosféricos disponibles en la actualidad nos pueden ayudar a entender el funcionamiento de la atmósfera, pero también el clima de distintos puntos de la superficie de Marte, en especial de aquellos a los que se planea enviar misiones robóticas. Por un lado, éstas se verán influenciadas por dichas condiciones climáticas, y, por el otro, permitirá seleccionar el tipo de materiales y tecnología que se adapten a las condiciones climáticas a las que serán expuestos los diferentes robots durante su vida útil en la superficie marciana.

Por este motivo, en este trabajo buscamos conocer qué información se puede extraer de los datos atmosféricos disponibles de Marte, y saber si en el futuro se podrán aplicar a las zonas de amartizaje de futuras misiones al planeta rojo. Para ello, comenzaremos dando una descripción detallada del comportamiento de la atmósfera de Marte (comparada con la Tierra) para conocer su dinámica, y a continuación evaluamos los datos disponibles en la Base Climática de Marte (Forget et al., 1999; Lewis et al., 1999). Este estudio lo centraremos en un lugar concreto, la región de Nili Fossae, en el hemisferio Norte de Marte, que fue uno de los candidatos para el amartizaje de la misión Mars Science Laboratory (cuyo robot lleva como nombre Curiosity), de la agencia NASA.

\section{LA ATMÓSFERA DE MARTE}

La atmósfera de Marte tiene un espesor y una densidad mucho menores que las de la Tierra. La presión superficial media en Marte es una centésima parte de la terrestre, 
unas 0,007 atm (aproximadamente 7 mbar; Barlow, 2008). Además se producen importantes variaciones en la presión superficial, tanto debidas a las diferencias orográficas, como estacionales. Mientras que la presión sobre la cima de Olympus Mons (el mayor volcán de Marte y también del Sistema Solar) es de 0.7 mbar, en lo más profundo de Hellas (una gran cuenca de impacto) se pueden alcanzar 14 mbar. Estacionalmente, el ratio de variación es del $25 \%$ con un esquema similar año tras año, y que es debido a la variación en la concentración de dióxido de carbono $\left(\mathrm{CO}_{2}\right)$ y vapor de agua en la atmósfera (Leovy, 2001).

La atmósfera de Marte se compone esencialmente de $\mathrm{CO}_{2}$ y pequeñas cantidades de nitrógeno y argón, además de otros elementos traza (Barlow, 2008). También está formada por una delgada capa de ozono, que al ser 1.000 veces menor que en la Tierra, no bloquea tan eficazmente como en nuestro planeta los rayos ultravioleta (UV). Estas características confieren a la atmosfera marciana un calor específico reducido. Por ello, debido a su alta transparencia, la absorción de radiación por la atmósfera es muy reducida, tanto la emitida por la superficie como la solar incidente.

Los cambios de presión atmosférica, junto con el peculiar comportamiento orbital del planeta (el periodo orbital es prácticamente el doble que el de la Tierra y su órbita tiene la mayor excentricidad de todo el Sistema Solar) conllevan que las temperaturas en la superficie de Marte tengan importantes variaciones estacionales y latitudinales. La temperatura media superficial es de aproximadamente $218 \mathrm{~K}$ (Barlow, 2008), unos $-55^{\circ}$ C. Pero mientras que en el polo sur en invierno se alcanzan temperaturas de hasta $-140^{\circ}$ C, durante el día en latitudes medias se pueden alcanzar hasta los $22^{\circ} \mathrm{C}$ (ej., Kieffer et al., 1977; Anguita, 1998).

Además de las diferencias de temperaturas estacionales y latitudinales, la amplitud térmica diaria es muy elevada. Durante las tardes de verano del hemisferio sur se pueden alcanzar temperaturas superficiales de alrededor de $20^{\circ} \mathrm{C}$, pero al caer la noche se pueden descender a $-100^{\circ} \mathrm{C}$ en el mismo lugar (Forget et al., 2006). A lo largo del día, la superficie del planeta se calienta por absorción de radiación solar e intercambia calor con la atmósfera circundante básicamente a través de procesos de convección. La convección es un proceso mediante el cual se produce el movimiento de un fluido, en este caso los gases de la atmósfera, a favor de un gradiente de densidad (de Pater y Lissauer, 2001). Estos movimientos convectivos, pueden producir tornados de arena, de los que se hablará más adelante. Durante la noche, al cesar este calentamiento, la temperatura decrece rápidamente en las proximidades de la superficie y el vapor atmosférico se condensa, produciendo neblinas y brumas. A esto se une que la inercia térmica del suelo marciano sea muy pequeña comparada con la terrestre sumado a su bajo contenido en agua, teniendo una considerablemente menor capacidad de retener el calor. Esto está causado, en parte, por la sequedad del ambiente marciano.

Como es de suponer, por las características anunciadas anteriormente, la estratificación de la atmósfera marciana es también diferente a la terrestre. La atmósfera marciana puede dividirse en tres partes (superior, media e inferior). Cuando se encuentra despejada (es decir con poca densidad de polvo en suspensión), en la parte inferior la temperatura disminuye con la altura (al igual que en la troposfera terrestre). Esta capa alcanza aproximadamente $45 \mathrm{~km}$ de altura. A partir de aquí, en la parte media de la atmósfera, la tendencia es similar. No se produce la inversión térmica que se da en la estratosfera terrestre, dado que la cantidad de ozono es insuficiente para a absorber la radiación UV. El gradiente negativo en esta zona se debe a la absorción de energía en el infrarrojo cercano y la emisión de radiación por parte del $\mathrm{CO}_{2}$, al igual que en la mesosfera terrestre (Schofield et al., 1997). Ya en la parte superior, a partir de los 110 $\mathrm{km}$ de altura, el gradiente se invierte. Aumenta la temperatura con la altitud, como en la termosfera de la Tierra. Esto se debe también a que el efecto de la absorción de la 
radiación UV aumenta con la altura. A partir de $125 \mathrm{~km}$ de altura, los gases atmosféricos comienzan a separarse por difusión a causa de las radiaciones ionizantes, permitiendo que las moléculas más volátiles escapen al espacio. Eso se produce dado que el campo gravitatorio que hay a esta altura, no es capaz de mantener a estas partículas dentro de su influencia. Esto explica el enriquecimiento atmosférico de isotopos pesados, como el deuterio (Carr, 2006).

Lo anteriormente descrito se da en condiciones de gran transparencia atmosférica, cuando la atmosfera se encuentra prácticamente libre de partículas en suspensión. Sin embargo, en Marte se suceden importantes tormentas de arena a escala planetaria. En estas condiciones, la transferencia de calor sobre la superficie decrece de manera importante, ya que las partículas en suspensión absorben la mayor parte de la radiación incidente sobre el planeta. Por la noche, estas partículas emiten radiación infrarroja que calienta la superficie (Forget et al., 2006). De esta forma, a mayor cantidad de partículas, el gradiente vertical de temperaturas es más isotérmico y las variaciones de temperatura diurnas se ven atenuadas (Carr, 2006). La cantidad de partículas en suspensión es mucho mayor que en la Tierra, dado que la lluvia y la nieve son mucho menos frecuentes y eficientes en Marte.

Al igual que en la Tierra, la temperatura de la atmósfera marciana desciende hasta alcanzar el punto de condensación y congelación de algunas moléculas. Las finas gotas y cristales que se producen entonces pueden formar nubes. En las regiones ecuatoriales, a una altitud de $10 \mathrm{~km}$, aparecen nubes de agua y hielo de color blanco (de Pater y Lissauer, 2001). Muchas son de naturaleza orográfica, produciéndose la condensación de este vapor al elevarse la columna de aire sobre relieves pronunciados, como en la región de Tharsis (Barlow, 2008). En el polo, donde las temperaturas son considerablemente menores y no superan el punto de congelación del agua, se forma una permanente cubierta polar formada por nubes de hielo. Las condiciones de presión en Marte hacen que el $\mathrm{CO}_{2}$ solo pueda estar en estado sólido o gaseoso (su temperatura de sublimación es de unos $-78,6^{\circ} \mathrm{C}$ ). De esta forma, en altitudes de unos $50 \mathrm{~km}$ sobre la superficie se encuentran nubes de hielo de $\mathrm{CO}_{2}$ (de Pater y Lissauer, 2001). Un último tipo de nubes de color amarillento, corresponden a las ocasionadas por tormentas de arena al elevar partículas en suspensión (Barlow, 2008).

Las nubes, además, pueden variar la temperatura superficial y la estructura atmosférica de manera considerable. Reducen la radiación incidente, ya que son muy reflectivas, y además bloquean la radiación infrarroja emitida por la superficie, aumentando el efecto invernadero.

La circulación atmosférica en Marte se rige principalmente, al igual que en la Tierra, por los contrastes térmicos de su superficie ( $y$, por tanto, de presión) y la rotación del planeta. Las variaciones térmicas producen corrientes convectivas y están provocadas por varios factores. Uno de ellos es el cambio estacional resultante de la condensación y sublimación de $\mathrm{CO}_{2}$ y $\mathrm{H}_{2} \mathrm{O}$ en las zonas polares. Otros son las tormentas de arena y las diferencias de temperatura entre la parte iluminada del planeta y la que no lo está (Barlow, 2008). El aire caliente se expande y se eleva provocando corrientes termales, de las zonas calientes (que también se encuentran a mayor presión). Debido a esto, los vientos a escala planetaria siguen el modelo de circulación aproximado de las células de Hadley, descritas para la Tierra (de Pater y Lissauer, 2001; Barlow, 2008). Las masas calientes de aire se mueven a mayor altitud hacia las zonas frías y al enfriarse vuelven a descender. Así se desplazan a las zonas más cálidas, repitiendo un ciclo de circulación meridional. En lugar de las dos células de Hadley teóricas que encontramos en la Tierra (una por hemisferio), en Marte se reduciría a una gran célula mucho más desarrollada en altitud por la falta de estratosfera, y que opera en ambos hemisferios (Forget et al., 2006). La célula se vería modificada por la rotación, oblicuidad, excentricidad orbital y la 
presencia de casquetes polares. Los dos últimos factores producen variaciones anuales de presión de hasta el $20 \%$. Durante los equinoccios la célula es simétrica, pero en los solsticios, la corriente ascendente se desplaza $30^{\circ}$ en el hemisferio de verano y la descendente $60^{\circ}$ en el hemisferio de invierno (Haberle et al., 1993) y las corrientes son más fuertes durante el invierno del hemisferio septentrional (Barlow, 2008).

La unión de la circulación meridional con la rotación del planeta (fuerza de Coriolis), da lugar a unos vientos de componente este y oeste (vientos zonales o trade winds), que dominan en la circulación general atmosférica de Marte (Forget et al., 2006). La similitud entre las características orbitales de Marte y la Tierra hacen que sus elementos meteorológicos sean muy similares. Se producen vientos zonales provenientes del este en latitudes medias todo el año, exceptuando a mediados de verano, que provienen del oeste. En latitudes bajas los vientos provenientes del oeste son los dominantes durante todo el año. En los trópicos, la célula de Hadley produce vientos del noreste en el hemisferio norte y del sudeste en el sur, el análogo a los vientos alisios terrestres. Sin embargo, los cambios estacionales en esta corriente, hacen que estos vientos se produzcan en latitudes medias durante el invierno (Barlow, 2008). En las regiones subtropicales (entre $\operatorname{los} 15^{\circ}$ y $\operatorname{los} 30^{\circ} \mathrm{N}$ y S), durante el solsticio, se produce una corriente en chorro (jet-stream) procedente del oeste con velocidades de hasta 33 $\mathrm{m} \cdot \mathrm{s}^{-1}$ a $2 \mathrm{~km}$ de altitud (Hinson et al., 1999), mientras que los vientos en superficie suelen encontrarse entre los 5 y los $10 \mathrm{~m} \cdot \mathrm{s}^{-1}$ (Joshi et al., 1995). En los polos se produce el equivalente a los vientos catabáticos terrestres, que salen desde los polos (a causa de las diferencias de presión y temperatura) y divergen latitudinalmente a causa de la rotación planetaria.

Como se ha mencionado anteriormente, los movimientos convectivos en la atmosfera, pueden producir tornados de arena (dust devils) (Greeley et al., 2006). Estos tornados pueden alcanzar diámetros de varios kilómetros y hasta unos $10 \mathrm{~km}$ de altura (la convección alcanza estas cotas ante la ausencia de estratosfera), cincuenta veces la anchura y diez veces la altura de sus análogos terrestres (Smith y Renno, 2001). Estos grandes tornados ponen en suspensión parte del polvo superficial, pequeñas partículas de aproximadamente $100 \mu \mathrm{m}$ (Forget et al., 2006). Este material en suspensión puede formar tormentas de polvo (Basu et al., 2004; Kahre et al., 2007), a escala local, regional o planetaria (Barlow, 2008). Las tormentas suelen darse con mayor frecuencia en el hemisferio sur.

La estacionalidad de los polos afecta de forma considerable a la atmósfera, especialmente a los ciclos del $\mathrm{H}_{2} \mathrm{O}$ y el $\mathrm{CO}_{2}$. Durante el verano, en ambos hemisferios se produce la sublimación de parte de sus polos. En el del hemisferio norte, la superficie de los polos se calienta, sublimándose completamente la capa de hielo seco $\left(\mathrm{CO}_{2}\right)$ y parte del hielo de agua. La circulación atmosférica transporta estos gases a otras latitudes incrementando levemente la presión atmosférica del planeta (Forget et al., 2006). Sin embargo, en el polo sur, la superficie no se calienta lo suficiente y se sublima únicamente parte del hielo de $\mathrm{CO}_{2}$. Así, parte del $\mathrm{H}_{2} \mathrm{O}$ que se sublima en el verano septentrional, en lugar de volver a solidificarse en este hemisferio, pasaría a engrosar la escasa cantidad de hielo de agua en el polo meridional. Obviamente esta dinámica no ha podido funcionar de esta forma durante toda la historia marciana, por lo que se supone que el ciclo operó de forma inversa en el pasado (Forget et al. 2006).

El ciclo del $\mathrm{CO}_{2}$ (James et al, 1992), está altamente conectado con el ciclo del polvo, ya que las corrientes ascendentes asociadas a la sublimación, transfieren polvo de la capa de hielo a la atmósfera, mientras que las corrientes descendentes depositan polvo sobre las capas polares (Kahn et al., 1992; James et al., 1999). En ciclo del $\mathrm{H}_{2} \mathrm{O}$ marciano se producen intercambios entre los reservorios por una parte la atmosféra y por otra las capas polares (permanentes y estacionales) y el agua adsorbida en el 
regolito y el hielo (superficial y subsuperficial). El transporte se produce principalmente como vapor de agua y en menor medida como nubes blancas de condensación. La

concentración de $\mathrm{H}_{2} \mathrm{O}$ en la atmósfera también tiene variaciones latitudinales y estacionales, causadas por la sublimación y condensación en las capas polares, desorción de agua por el regolito (ante los cambios de temperatura estacionales) y la difusión del vapor del regolito a la atmósfera. La mayor concentración de vapor se produce entre primavera/verano y la menor en otoño/invierno. (Barlow, 2008).

Por tanto, una vez descritos las características generales de la atmosfera de Marte, pueden estudiarse más en detalle algunas de ellas mediante datos cuantitativos. Para ello nos deberemos centrar en una zona adecuada, que sea representativa. En el siguiente apartado se describe y justifica la zona de estudio elegida.

\section{ZONA DE ESTUDIO}

Con el fin de realizar un estudio que permita obtener una idea general de la atmósfera de Marte, ha sido necesario seleccionar un área del hemisferio norte, cercana a la dicotomía (el límite neto entre las Tierras Bajas al norte, y las meridionales Tierras Altas), para ser representativa de todo el planeta. Esto es así porque sería un punto medio entre. Además esta zona debería encontrarse alejada de los grandes relieves, para que estos no influyeran en el comportamiento atmosférico regional, esto es, evitando el complejo volcánico de Tharsis, así como las dos grandes cuencas de impacto de Hellas y Argyre (Figura 1). Por todo lo anterior, la zona de Nili fosase, al oeste de Isidis planitia y al norte de Syrtis Major, que se localiza en las coordenadas $21.00^{\circ} \mathrm{N}, 74.45^{\circ} \mathrm{E}$ (Figura 1 ), nos ha parecido una zona muy adecuada para llevar a cabo el estudio de la variabilidad anual y diaria de temperatura, presiones y otras características atmosféricas de interés.

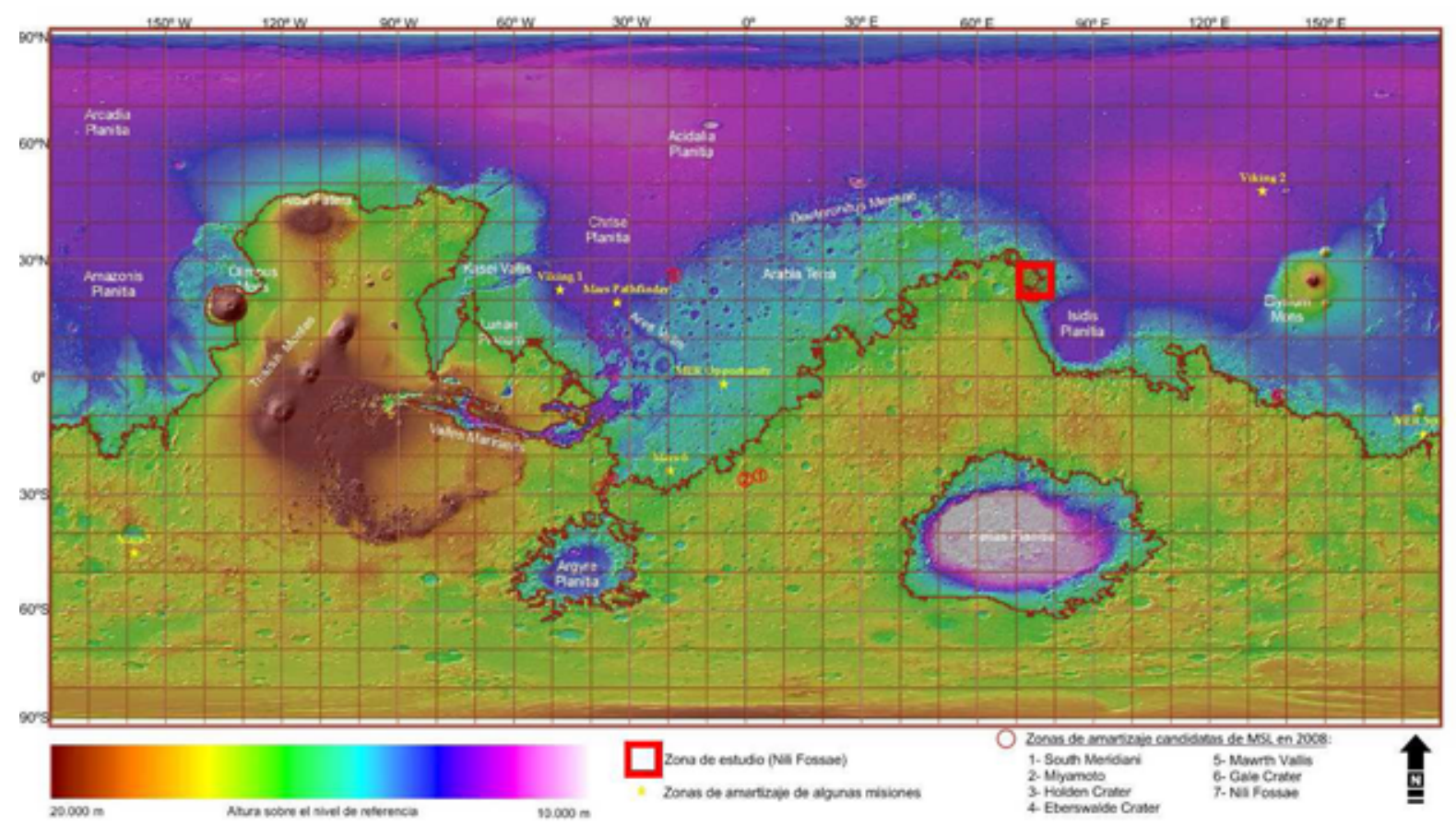

Figura 1. Localización de la zona de estudio (recuadro rojo) sobre la topografía general del planeta. Se muestran algunos lugares geográficos destacados (etiquetas blancas), zonas de amartizaje de algunas misiones y las zonas de amartizaje candidatas de la misión Mars Science Laboratory (MSL) que se barajaban en 2008. La línea negra representa el nivel de referencia: altitud 0m. 


\section{DATOS Y MÉTODOS}

La Base de Datos Climática de Marte (Mars Climate Database, MCD; Forget et al., 1999; Lewis et al., 1999) es una base de datos de los parámetros que describen el clima y el medio ambiente de la atmósfera de Marte. Está construida basándose en las integraciones multianuales obtenidas del Modelo Climático Global (Global Climate Model, MCG), desarrollado por el Laboratoire de Météorologie Dynamique du CNRS (Francia), en colaboración con la Universidad de Oxford (Reino Unido), el Instituto de Astrofísica de Andalucía (España), y SA (Francia), con el apoyo de la Agencia Espacial Europea (European Space Agency, ESA) y el Centre National d'Etudes Spatiales (CNES). Este modelo de circulación atmosférica, se basa en las leyes físicas que explican el funcionamiento de los parámetros que la gobiernan y las condiciones de intercambio energético en la superficie del planeta, y ha sido validado exhaustivamente mediante datos observacionales de las misiones Viking, Mars Global Surveyor (MGS) y Mars Odyissey (MO) (Millour y Forget, 2008).

Los datos de la MCD se encuentran ordenados en 12 meses tipo para el año marciano, que tienen una duración de $30^{\circ}$ de longitud solar (Ls) dada uno (se divide la órbita alrededor del Sol y se comienza a contar desde el equinoccio de primavera septentrional, de forma que los meses se encuentran centrados en $L s=15^{\circ}, 45^{\circ}$, etc.). Debido a la excentricidad de la órbita de Marte alrededor del Sol, la duración de los meses en el calendario en Marte varía entre los 46 y los 66 soles (o días marcianos). Las horas están definidas como $1 / 24$ de un sol, mostrándose en los gráficos únicamente cada dos horas, y correspondiendo a la hora solar real (el Sol se encuentra en su posición más alta al medio día) en el meridiano $0^{\circ}$. La resolución espacial de los datos es de unos $3.75^{\circ}$ en latitud y $5,625^{\circ}$ en longitud (Forget et al., 1999).

Como el factor que más condiciona el comportamiento de los parámetros atmosféricos en Marte es la cantidad y distribución de las partículas en suspensión y dado que los detalles sobre la distribución de polvo atmosférico son impredecibles, en la MCD se han tomado tres escenarios distintos, en función de la cantidad de polvo en suspensión. El escenario medio (Mars Year 24) se basa en las condiciones que reunía el planeta en el momento en que fue observada por la Mars Global Surveyor (MGS), entre los años terrestres 1999 y 2001 . El nombre de este escenario se debe a que corresponde al año marciano número 24, según el calendario propuesto en Clancy et al. (2000). Los otros dos escenarios, se denominan "cálido" y "frío". El primero corresponde a una atmósfera extremadamente clara, con muy poco polvo en suspensión que bloquee las radiaciones solares, y por tanto con mayor temperatura en la atmósfera. El escenario frío, sin embargo, supone unas condiciones de mayor opacidad, ocasionadas previamente a la formación de tormentas de arena, condiciones que se dan únicamente durante el otoño e invierno del hemisferio norte (Ls entre el $180^{\circ}$ y el $360^{\circ}$ ). Un último escenario contemplaría un planeta totalmente cubierto por una tormenta de polvo global.

Como ya se ha comentado, en este trabajo se analizan múltiples variables meteorológicas y térmicas, tales como la temperatura de la superficie del suelo y la atmosférica, la presión atmosférica a nivel de la superficie del suelo y los flujos energéticos entrantes y salientes de radiación solar (SWR) e infrarroja (IR). Con el fin de obtener una idea general del clima en el punto estudiado, de todos los datos disponibles en la base de datos, se han usado únicamente los datos correspondientes a las condiciones climáticas medias (año marciano número 24), tomando datos representativos para estudiar variabilidades anuales y diarias. También se estudia en este trabajo el perfil altitudinal de temperaturas de la atmósfera en la zona de estudio, es decir, la variación de temperatura que se produce con la altura. Para comprender la 
distribución especial de los datos, se han realizado mapas de todo el planeta que representan cada uno de los parámetros estudiados (temperatura de la superficie del suelo, temperatura y presión atmosférica, flujos de radiación...), tomando diversas combinaciones de meses, horas y alturas. En el caso de los gráficos, se ha tomado el dato de $22,50^{\circ} \mathrm{N}$ y $73,12^{\circ} \mathrm{E}$ (Figura 1 ), que es el dato disponible más cercano al área de estudio.

\section{TEMPERATURA SUPERFICIAL}

La temperatura de la superficie del suelo en la zona de estudio es importante para estudiar la presencia de agua líquida en superficie y bajo ella, y depende tanto de los materiales como de la exposición de la misma a la radiación solar (ángulo de incidencia, intensidad, opacidad atmosférica, etc.).

En la Figura 2, se presenta la variación de la temperatura superficial estimada para el planeta a lo largo del año marciano. Las imágenes corresponden al día medio de cada mes durante el día (a las $8 \mathrm{~h}$ ) y durante la noche (a las 20h) en la zona de estudio. Lo que se aprecia en esta figura es la oscilación de la zona donde inciden los rayos solares, localizándose más al norte durante el mes 4 y más al sur durante el mes 10, meses donde la inclinación del eje es máxima. La radiación solar está relacionada directamente con los valores de temperatura superficial, localizándose el máximo donde estos inciden perpendicularmente. Se registran unas temperaturas mínimas de $143 \mathrm{~K}$ y máximas de $312 \mathrm{~K}$ a lo largo de todo el planeta. Observando las imágenes (Figura 2), también se aprecia que los valores mínimos se localizan en los polos y en las regiones de Tharsis y Elysium. En estas últimas, al ser zonas de mayor elevación, presentan una menor temperatura por el efecto de la topografía. El polo norte estará más frío entre los meses 7 y 2 , mientras que el sur lo estaría entre el 1 y el 8 , alcanzando este último unas temperaturas inferiores.

En las imágenes correspondientes al día, la región de Tharsis (Tharsis Montes, Figura 1) presenta menores temperaturas, tanto por encontrarse en el lado opuesto del planeta donde se produce la incidencia normal de los rayos solares, como por su mayor elevación. Parece que sobre las Tierras Altas se registran las temperaturas más altas. En las inmediaciones de la zona de estudio los valores más elevados se alcanzan en los meses 6 y 7. En las imágenes de noche, se aprecian otras dos zonas destacadas en las zonas de menor insolación. Una de ellas, al Este, corresponde al domo de Elysium (Elysium Mons, Figura 1), debido a su mayor elevación (al igual de Tharsis). La otra se localiza en Arabia Terra (Figura 1), cercana a la zona de estudio.

Para entender mejor el comportamiento de estos datos se ha realizado un gráfico con los datos más cercanos a la zona de estudio (Figura 2, gráfica bajo mapas). Los valores medios para el día son de aproximadamente $273,7 \mathrm{~K}\left(0,6^{\circ} \mathrm{C}\right)$ y $190,1 \mathrm{~K}$ durante la noche $\left(-83^{\circ} \mathrm{C}\right)$. Se aprecia que la variación interanual es mayor durante el día que durante la noche (un rango de amplitud térmica de $27 \mathrm{~K}$ ), con un máximo en el mes 6 y un mínimo en el 9. Estos valores máximos y mínimos se repiten durante la noche, pero con una menor amplitud térmica $(8,7 \mathrm{~K})$. En estos datos se observa cómo, aunque la variación que se produce en la temperatura superficial a una misma hora a lo largo del año, no es muy grande; no ocurre lo mismo con la amplitud térmica entre el día y la noche. 


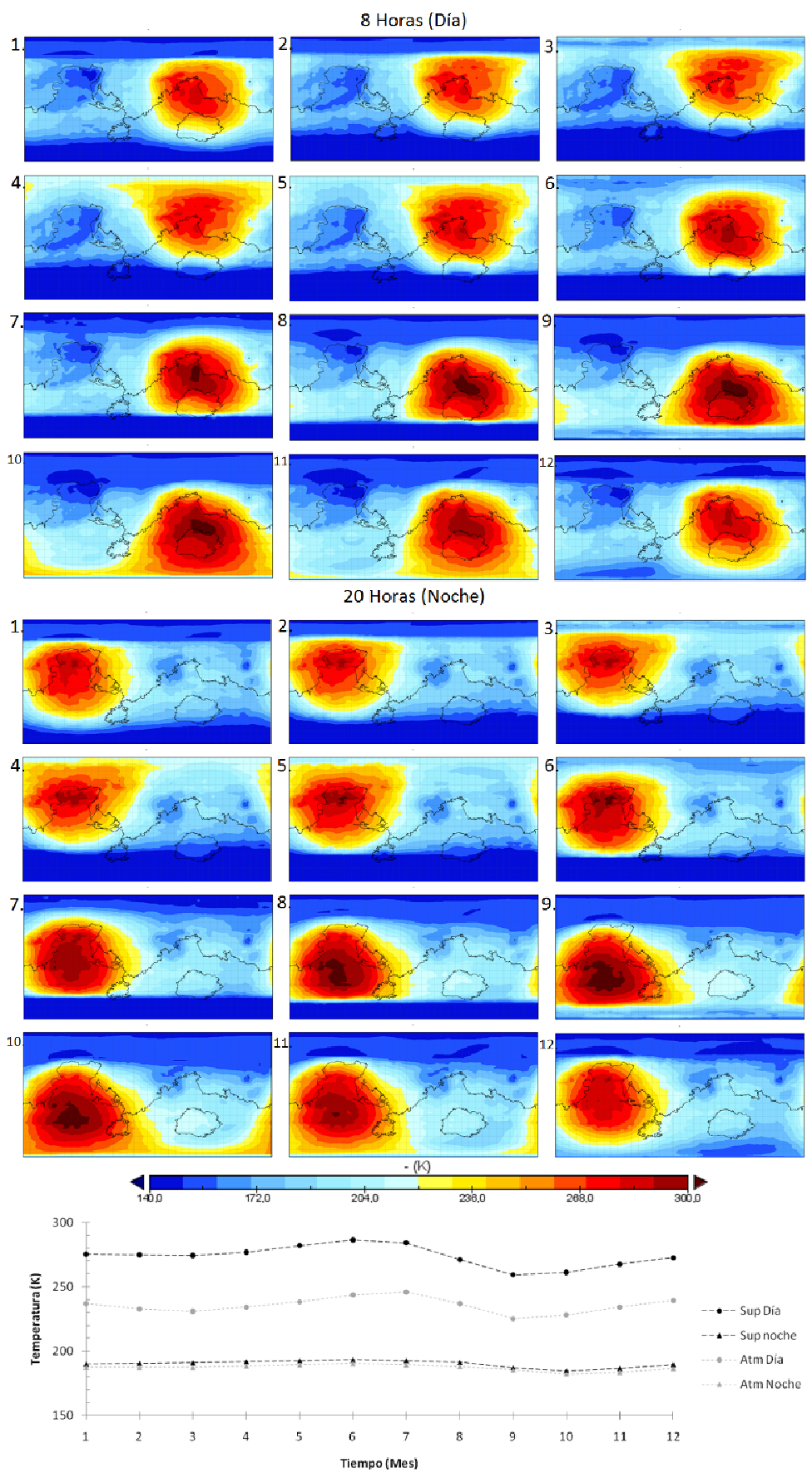

Figura 2. Mapas de distribución de temperatura superficial en Marte, a las $8 \mathrm{~h}$ (arriba, día en la zona de estudio) y a las $20 \mathrm{~h}$ (abajo, noche en la zona de estudio), durante los 12 meses un año medio. La línea negra representa el nivel de referencia: altitud $0 \mathrm{~m}$. Abajo, se muestran estos datos para la zona de estudio (negro), así como los de temperatura atmosférica a $5,53 \mathrm{~m}$ para el día $(12 \mathrm{~h})$ y la noche $(00 \mathrm{~h})$. Datos obtenidos a partir de la Base de Datos Climática de Marte (Forget el al., 1999; Lewis et al., 1999). 
Para conocer mejor la naturaleza de esta variabilidad térmica diaria se han tomado las temperaturas de la superficie del suelo cada dos horas a lo largo del día, en los meses con valores extremos, es decir, los meses 6 y 9 (Figura 3). Al igual que en la Figura 2, las diferencias más importantes se dan entre las zonas favorables a la incidencia de la radiación solar y las que no. En este caso la variación se da respecto a la trayectoria solar respecto de la superficie del planeta, desplazándose las zonas de mayor temperatura superficial hacia el Este conforme transcurre el día. También se ha realizado un gráfico para conocer el comportamiento en la zona de estudio (Figura 3, abajo), tomando días medios en cuatro meses distintos (1, 4, 7 y 10). Aquí se puede observar cómo se produce un gran incremento de las temperaturas entre las 2 y las 6 horas, alcanzando un máximo a las $8 \mathrm{~h}$ de hasta $283,9 \mathrm{~K}\left(10,8^{\circ} \mathrm{C}\right)$, para descender rápidamente desde las 10 hasta las 16 horas. Desde las 16 a las $0 \mathrm{~h}$, las temperaturas no variarían en gran medida, simplemente descenderían de un modo suave a causa del paulatino enfriamiento de la superficie, para volver a subir repentinamente al día siguiente con la salida del Sol. Los valores medios más altos de la temperatura se obtienen para el mes $4\left(224,1 \mathrm{~K}\right.$, unos $\left.-49^{\circ} \mathrm{C}\right)$, aunque el mes 7 tenga un mínimo y un máximo superiores (con amplitud térmica de 97,6 K). Los meses 1,4 y 7 tienen valores muy similares, encontrando los inferiores durante el mes 10 , con 208,2 K de media ($\left.64,9^{\circ} \mathrm{C}\right)$ y un mínimo de $178,4 \mathrm{~K}\left(-94,8^{\circ} \mathrm{C}\right)$.

\section{TEMPERATURA ATMOSFÉRICA}

Otro dato que se puede obtener mediante la Base de Datos Climática de Marte $(M C D)$ es la temperatura atmosférica para varias alturas. En este trabajo se han estudiado con profundidad los registros a la menor altura posible $(5,53 \mathrm{~m})$, que son los que tienen más importancia para el estudio del intercambio energético en la superficie del suelo. Estas temperaturas, al ser comparadas con las superficiales, pueden ayudar a entender mejor las relaciones energéticas entre el suelo y la atmosfera, que son de gran interés en el estudio de materias relacionadas con la habitabilidad y el permafrost. También se ha realizado un perfil de temperaturas con la altura en la atmósfera para la zona de estudio. 

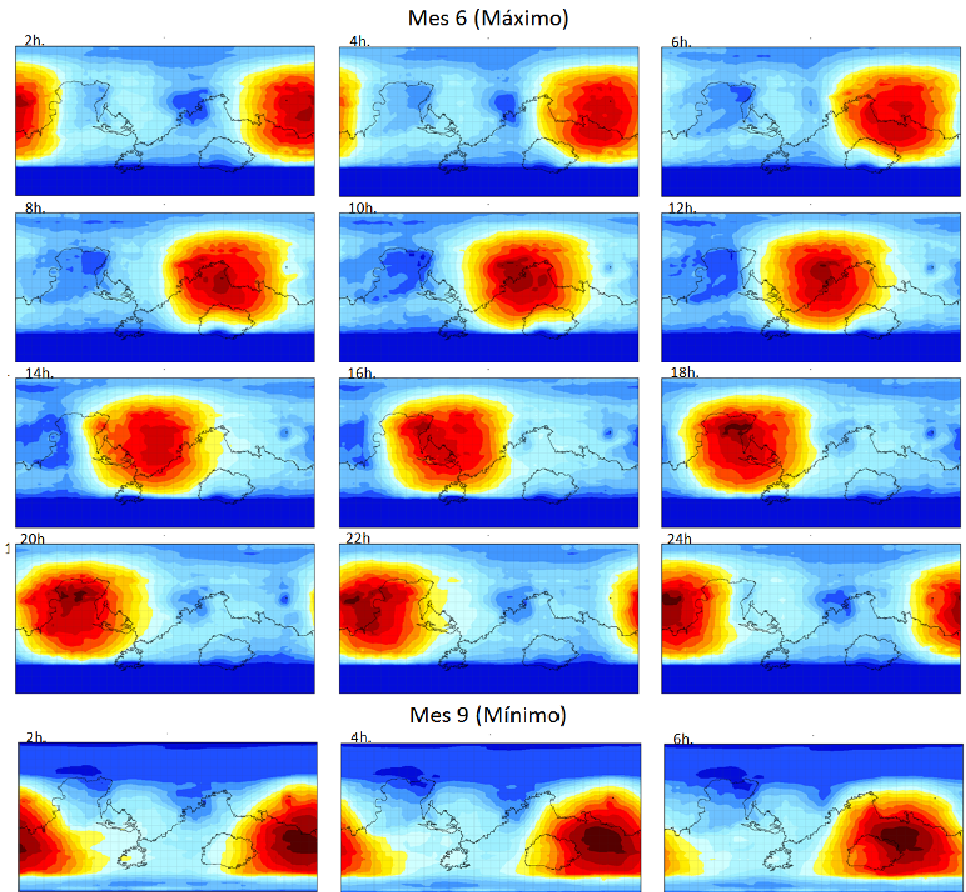

Mes 9 (Mínimo)
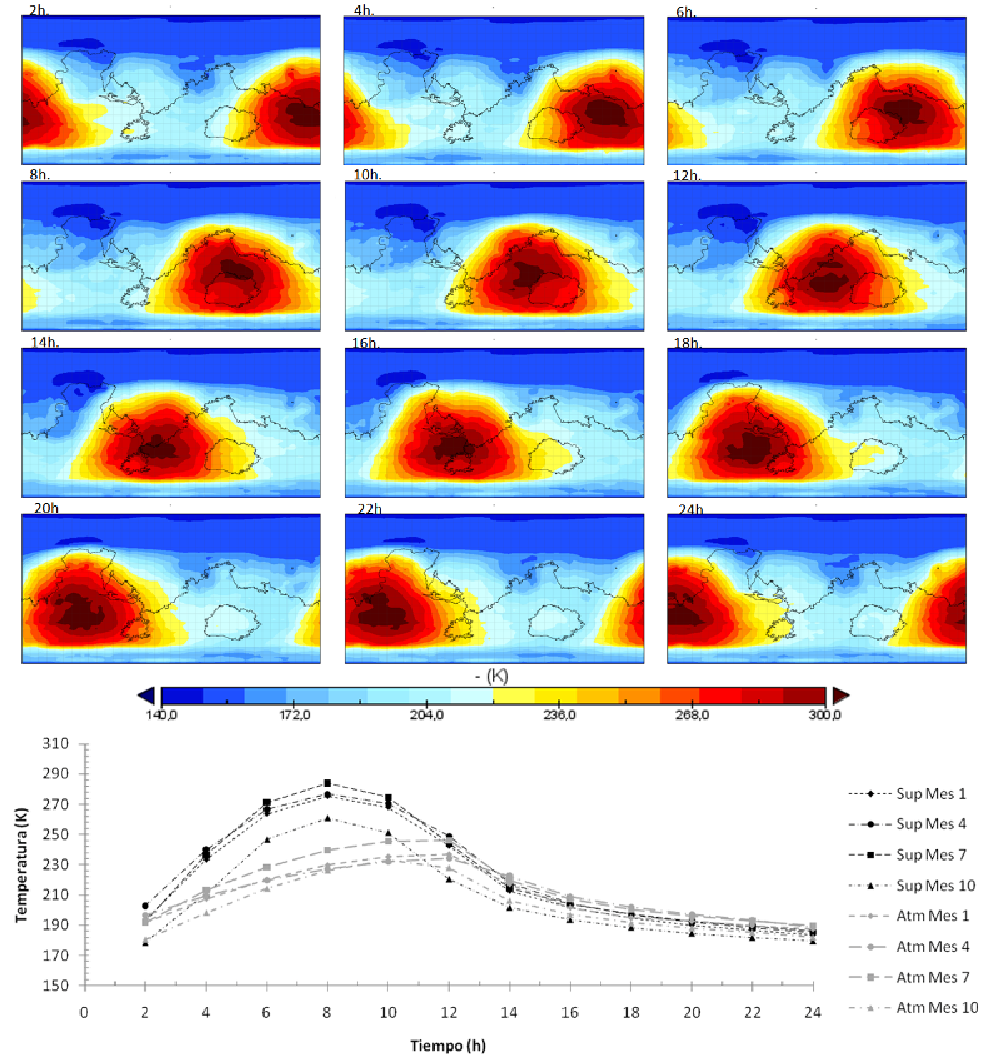

Figura 3. Mapas de de distribución de temperaturas superficiales de Marte, durante el mes 6 (arriba) y el 9 (abajo), cada dos horas. En estos meses se alcanza el máximo y el mínimo en la zona. La línea negra representa el nivel de referencia: altitud $0 \mathrm{~m}$. Abajo datos para la zona de estudio, junto con los de temperatura atmosférica a 5,53 m. Datos obtenidos a partir de la Base de Datos Climática de Marte (Forget el al., 1999; Lewis et al., 1999). 
Las variaciones de temperatura atmosférica a 5,53 metros (Figura 4), siguen un patrón similar a lo largo del año del que se ha podido observar en la temperatura superficial (Figuras 2 y 3 ). Por ello, para la variación anual se han tomado únicamente datos durante los meses $1,4,7$ y 10 , para el día (12 horas, cuando se produce el máximo en la zona) y la noche (00 horas, el mínimo). En los mapas (Figura 4, arriba) se aprecia como la zona de mayor temperatura no esta tan limitada a la radiación incidente como lo estaba en la temperatura superficial, aunque las temperaturas durante el día son considerablemente más bajas. También se producen similares anomalías en las regiones de Tharsis, Elysium y Arabia Terra (Figura 1). En los polos se registran unas temperaturas superiores, especialmente durante la noche (ver mes 1 y 7; Figura 4, arriba).

En el diagrama de la Figura 1 (abajo) se aprecia que al igual que con la temperatura superficial, la atmosférica a $5,53 \mathrm{~m}$ de la superficie también presenta una mayor amplitud durante el día $(20,7 \mathrm{~K})$ que durante la noche $(8,2 \mathrm{~K})$; siendo la de día $6,4 \mathrm{~K}$ menor, en este caso. Aunque la media de la temperatura atmosférica durante la noche es similar a la superficial $\left(187,1 \mathrm{~K}\right.$ o $\left.-86^{\circ} \mathrm{C}\right)$, la diurna es $38,2 \mathrm{~K}$ menor $(235,5 \mathrm{~K}$ o $\left.-37,6{ }^{\circ} \mathrm{C}\right)$. El máximo a las 12 horas se alcanza en el mes $7\left(245,9 \mathrm{~K} \mathrm{o}-27,3^{\circ} \mathrm{C}\right)$ y el mínimo en el mes $9\left(225,2 \mathrm{~K} \circ-48,0^{\circ} \mathrm{C}\right)$, de forma muy similar a la temperatura superficial.

Al igual que con las variaciones anuales, como las tendencias son también similares durante el día, se han tomado únicamente las 2, 8, 14 y 20 horas; también para los meses 6 y 9 (Figura 4, abajo) que son los meses con valores máximos y mínimos, respectivamente, y que permiten realizar una comparativa con los datos de temperatura superficial. Se observa como la temperatura atmosférica es mayor durante el mes 9 en todo el planeta, aunque debido a la inclinación axial, el área de mayor insolación y de mayor temperatura por tanto, se localiza más al norte, y más próxima a la zona de estudio. Las anomalías que se registran en Tharsis, Elysium y Arabia Terra producen áreas con mayores temperaturas. Este efecto provoca que, por ejemplo, durante el mes 6 a las $20 \mathrm{~h}$ las temperaturas en la zona sean mayores que en las inmediaciones. 
Día (12 h)
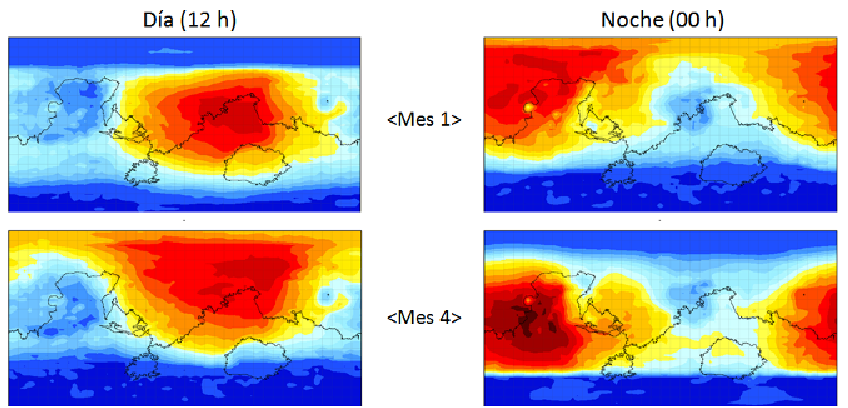

$<$ Mes 4>
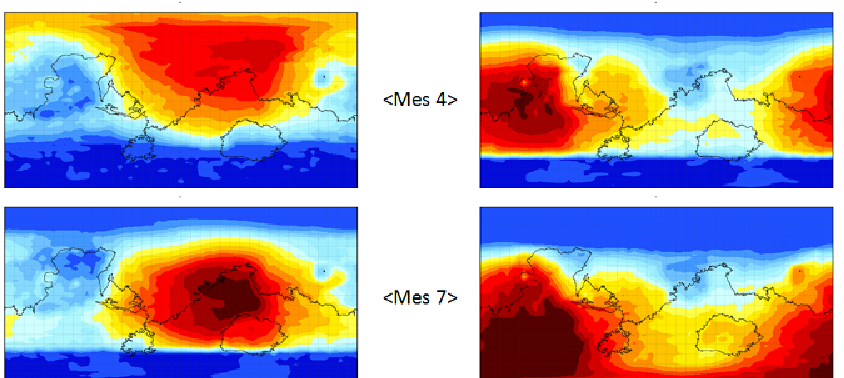

$<$ Mes 7>
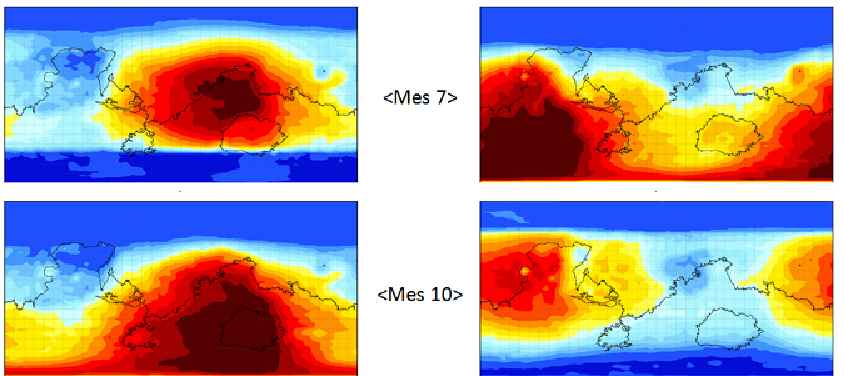

Mes 6 (Máx.)

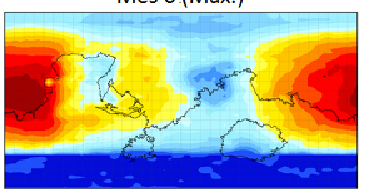

$<2 \mathrm{~h}>$

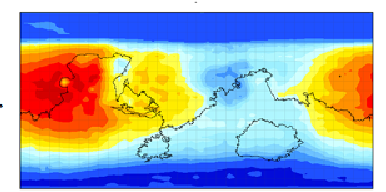

Mes 9 (Min.)
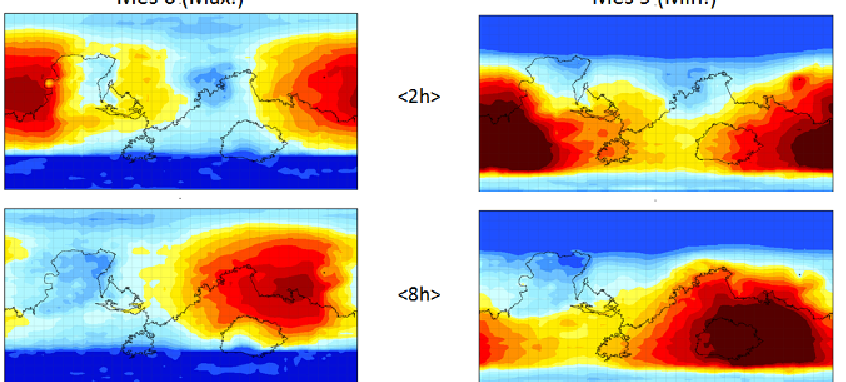

$<8 \mathrm{~h}$
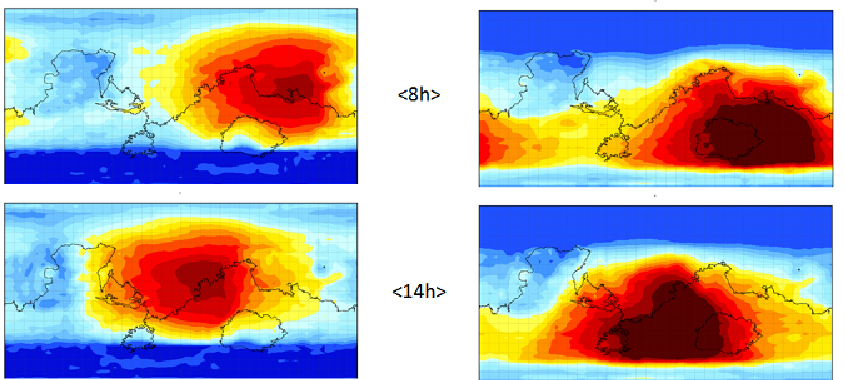

$<14 h>$
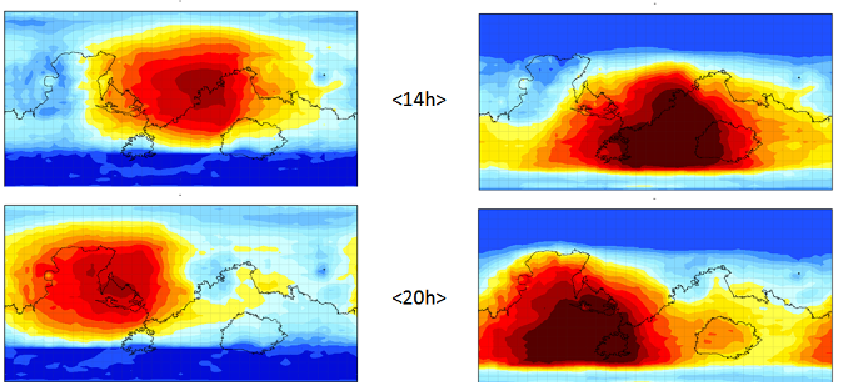

$<20 \mathrm{~h}>$

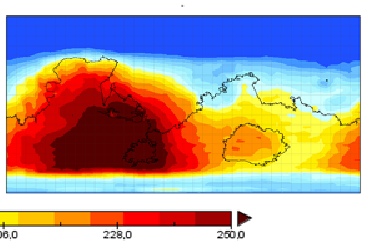

Figura 4. Mapas de temperatura atmosférica a 5,53 m en Marte, que muestra su distribución anual (Arriba, a las $12 \mathrm{~h}$ y a las $00 \mathrm{~h}$ durante los meses $1,4,7$ y 10) y diaria (Abajo, durante el mes 6 y el 9 en las horas 2, 8, 14 , y 20). La línea negra representa el nivel de referencia: altitud $0 \mathrm{~m}$. Datos obtenidos a partir de la Base de Datos Climática de Marte (Forget el al., 1999; Lewis et al., 1999). Los datos para la zona de estudio se representan en las Figuras 2 y 3 , junto a los de temperatura superficial. 
Para caracterizar la variación en la zona de estudio a lo largo del día marciano también se han incluido estos valores en una gráfica (Figura 3, abajo). Se aprecia como el máximo diario se produce en todos los meses a las 12 horas (236,3 K de media anual a esa hora, unos $-370 \mathrm{C}$ ). No hay gran variación de temperaturas entre los distintos meses, pero el más cálido parece ser el mes 7 , con un máximo de $356,9 \mathrm{~K}\left(-27^{\circ} \mathrm{C}\right)$ y unas temperaturas medias de $214,2 \mathrm{~K}\left(-59^{\circ} \mathrm{C}\right)$. Las temperaturas mínimas durante el día se producen a las 00 horas, con valores de $186,9 \mathrm{~K}$ de media anual $\left(-86^{\circ} \mathrm{C}\right)$, produciéndose los valores mínimos durante el mes 10 a las 2 horas $\left(180,7 \mathrm{~K} \mathrm{o}-93^{\circ} \mathrm{C}\right)$. La amplitud térmica es de aproximadamente $50 \mathrm{~K}$ durante el día y unos $10 \mathrm{~K}$ anuales.

Por último, se puede realizar un estudio de la distribución altimétrica de la temperatura a la que se encuentra la atmósfera sobre el área de estudio. Para ello, se han tomado los datos de los meses 1, 4, 7 y 10 para las alturas disponibles en la base de datos, hasta los $135 \mathrm{~km}$ de altitud. En la Figura 5 se muestran los valores de temperaturas de la columna resultante del estudio. Durante el mes 7 se producen las temperaturas más bajas (muy similares a las del mes 10, entre los $10 \mathrm{~km}$ y los $50 \mathrm{~km}$ ). Durante el mes 4, aparecen las temperaturas mayores durante los primeros $50 \mathrm{~km}$, a partir de ahí disminuyen pasando a ser las temperaturas más bajas, a partir de los 110 $\mathrm{km}$, aumentan de nuevo para volver a ser las más altas de la distribución altimétrica.

\section{PRESIÓN SUPERFICIAL}

La presión atmosférica es otro parámetro importante que podemos encontrar dentro de la Base de Datos Climática (Forget et al,. 1999; Lewis et al., 1999). Se seguirá el mismo procedimiento para su análisis que en apartados anteriores, analizando la presión a la mínima altitud disponible (5,53 m sobre la superficie del suelo).

La variabilidad a lo largo del día en la presión atmosférica no es muy significativa, por lo que se presentan los mapas para la hora en que se producen los valores mínimos de presión (12h) y para los que se dan los máximos (16h), y para cuatro meses $(1,4,7$, y 10). En la Figura 6, se puede ver el resultado y como los valores mayores corresponden a zonas deprimidas topográficamente, y los menores a las zonas de mayor elevación. La diferencia entre los valores de mañana y tarde, no se aprecian a simple vista. Se puede ver como durante el mes 4 las presiones son máximas en Hellas Planitia y mínimas en la región de Tharsis. Durante el mes 10 las máximas aparecen por todas las Tierras Bajas. 


\begin{tabular}{r|cccc}
\multirow{2}{*}{$\begin{array}{c}\text { Altitud } \\
(\mathrm{m})\end{array}$} & \multicolumn{4}{c}{ Meses } \\
\cline { 2 - 5 } $5,5,53$ & 236,9 & 234,2 & 245,9 & 228,0 \\
20,14 & 236,4 & 233,5 & 245,7 & 230,1 \\
49,56 & 236,2 & 233,5 & 245,5 & 231,1 \\
116,81 & 235,8 & 232,9 & 245,1 & 231,4 \\
253,40 & 235,0 & $\mathbf{2 3 2 , 1}$ & 244,2 & 231,0 \\
511,01 & 233,5 & 230,6 & 242,6 & 229,8 \\
992,13 & 230,6 & 227,8 & 239,7 & 227,5 \\
$1.859,23$ & 225,5 & 222,8 & 234,5 & 223,1 \\
$3.323,52$ & 217,3 & 214,6 & 226,0 & 220,6 \\
$5.605,41$ & 206,6 & 202,6 & 216,5 & 218,4 \\
$8.864,46$ & 199,3 & 193,4 & 209,8 & 213,4 \\
$13.136,86$ & 192,1 & 185,2 & 201,3 & 204,0 \\
$18.316,55$ & 184,4 & 175,6 & 195,2 & 196,1 \\
$24.195,64$ & 176,1 & 165,5 & 187,7 & 187,2 \\
$30.563,27$ & 166,5 & 154,9 & 177,2 & 177,0 \\
$37.287,65$ & 157,1 & 145,4 & 169,1 & 168,6 \\
$44.236,07$ & 152,4 & 142,0 & 164,5 & 164,0 \\
$51.236,03$ & 153,1 & 142,2 & 161,9 & 161,3 \\
$58.236,12$ & 155,2 & 142,5 & 161,2 & 157,0 \\
$65.236,12$ & 154,4 & 144,2 & 159,9 & 151,5 \\
$72.236,12$ & 148,4 & 148,8 & 153,9 & 146,8 \\
$79.236,12$ & 141,1 & 147,8 & $\mathbf{1 4 9 , 1}$ & 143,7 \\
$86.236,12$ & 135,5 & 147,6 & 145,3 & 141,3 \\
$93.236,12$ & 132,7 & 145,9 & $\mathbf{1 4 1 , 6}$ & 139,1 \\
$100.236,13$ & 139,9 & 143,1 & $\mathbf{1 4 3 , 3}$ & 137,9 \\
$107.236,18$ & 141,7 & 138,2 & $\mathbf{1 4 4 , 3}$ & 137,1 \\
$114.236,22$ & 137,4 & 133,6 & $\mathbf{1 4 2 , 6}$ & 134,7 \\
$121.236,22$ & 134,6 & 129,2 & $\mathbf{1 3 8 , 8}$ & 132,1 \\
$128.236,22$ & 132,3 & 123,9 & $\mathbf{1 3 4 , 9}$ & 129,9 \\
$135.236,22$ & 127,3 & 115,6 & $\mathbf{1 2 8 , 3}$ & 127,3
\end{tabular}

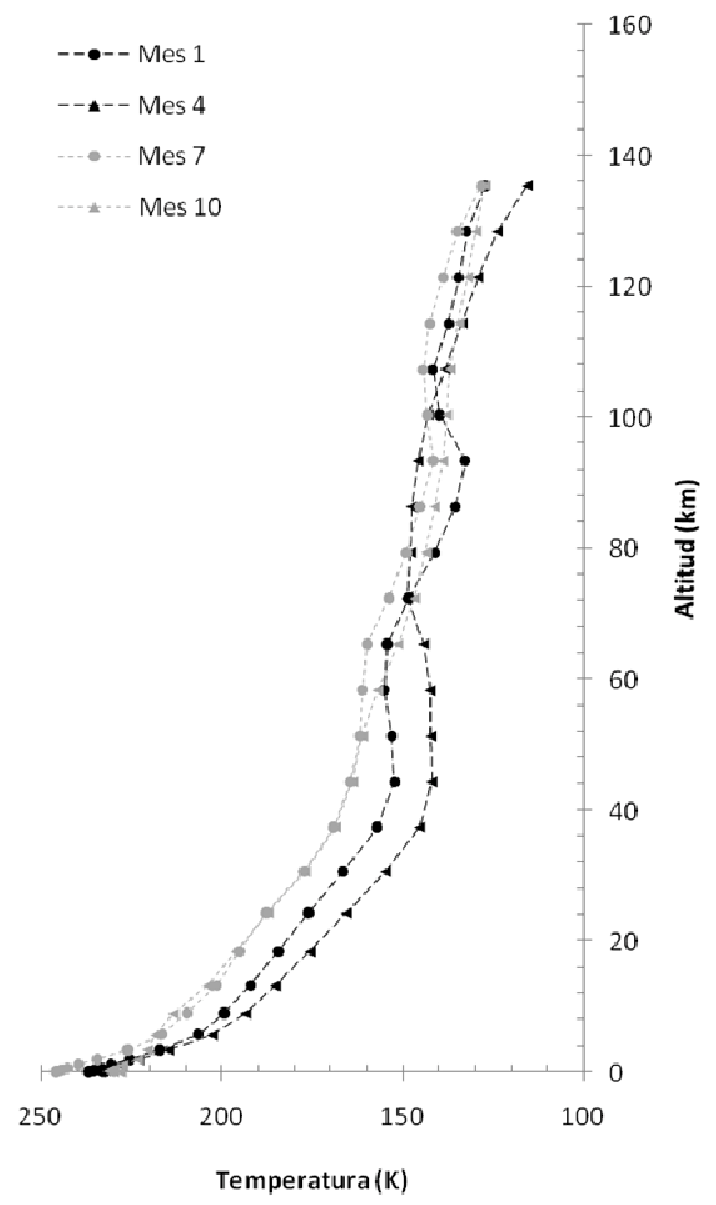

Figura 5. Tabla (izquierda) y gráfico (derecha) de variación de la temperatura atmosférica con la altura, en los meses 1, 4, 7 y 10, para la zona de estudio (Nili Fossae). Datos obtenidos a partir de la Base de Datos Climática de Marte (Forget el al., 1999; Lewis et al., 1999).

Obteniendo los datos para nuestra zona de estudio (Figura 6, arriba) se puede observar la gran diferencia de presiones que se produce a lo largo del año. Las presiones disminuyen del mes 3 al mes 6 bruscamente, alcanzando un mínimo de 498,7 Pa por la mañana y 505,3 Pa por la tarde. Las presiones se incrementan rápidamente, hasta un máximo en el mes 9 de 618,8 Pa por la mañana y 631,6 Pa por la tarde. En este intervalo las temperaturas de la mañana siguen una tendencia menos clara (Figura 6, arriba). Entre el mes 9 y el 3 del año siguiente, se produce un pequeño descenso y posterior incremento de las presiones. Estas diferencias de presión de 130 Pa se deben a la evaporación del $\mathrm{CO}_{2}$ y el $\mathrm{H}_{2} \mathrm{O}$ de los polos.

Como ya se ha comentado la variación de presión durante el día no es significativa, y no se aprecian diferencias considerables a simple vista. Por ello, no se muestran los mapas obtenidos y se publica directamente la tabla y el gráfico con los datos para los meses 1, 4, 7 y 10, cada dos horas (Figura 6, abajo). En el mes 10 se producen las mayores presiones medias $(606,1 \mathrm{~Pa})$, mientras que las menores se producen durante el mes 4 (550,2 Pa). La diferencia de presión anual es de unos 75-80 $\mathrm{Pa}$; siendo la diferencia diaria entre el máximo y el mínimo de $10 \mathrm{~Pa}$ aproximadamente. Durante el día, el máximo de presión se produce a las $16 \mathrm{~h}$ y el mínimo a las $12 \mathrm{~h}$ (exceptuando el mes 4 , que sigue un esquema de variación diferente). 


\section{FLUJOS DE RADIACIÓN}

Se muestran a continuación los flujos de energía solar (onda corta) y térmica (onda larga, IR), tanto hacia la superficie, como hacia el espacio. En la Figura 7 (arriba) se puede observar como el flujo de energía solar hacia la superficie tiene una estructura y comportamiento muy similar al de la temperatura superficial (Figuras 2 y 3 ), siendo máximo cuanto más perpendicular a la superficie es la incidencia de los rayos solares sobre la superficie. El flujo de energía solar al espacio (Figura 7, abajo), sin embargo, presenta una distribución menos regular y que está más relacionada con la topografía y la reflectividad de los materiales superficiales, como se aprecia por su comportamiento sobre Hellas y Argyre Planitia.
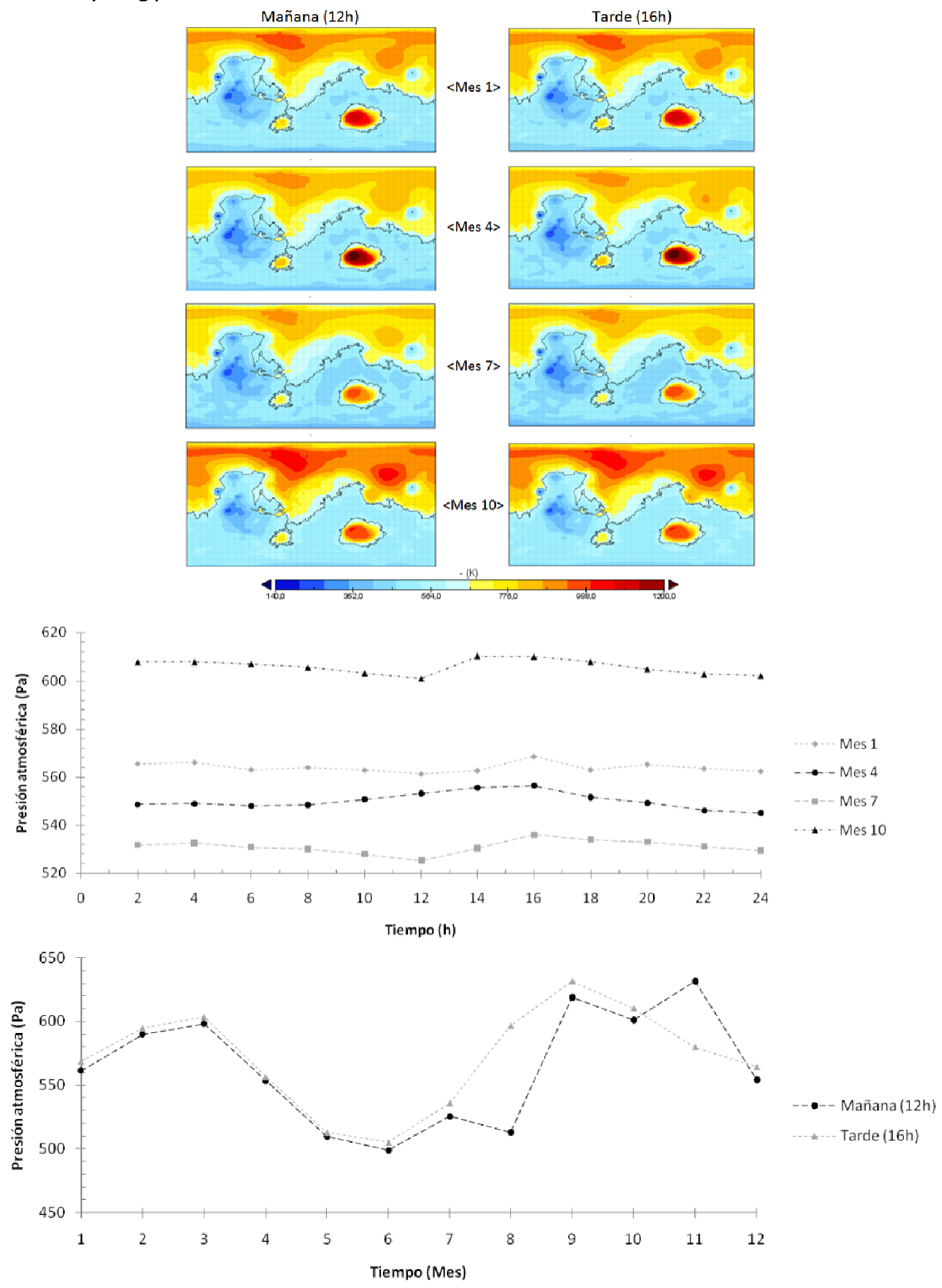

Figura 6. Mapas de presión atmosférica $(5,53 \mathrm{~m}$ sobre la superficie del suelo) en Marte, por la mañana (12h, izquierda) y por la tarde (16h, derecha), en los meses $1,4,7$ y 10 . La línea negra representa el nivel de referencia: altitud $0 \mathrm{~m}$ sobre la superficie del suelo. También se muestran dos gráficos que representan la variabilidad diaria (arriba) y anual (abajo) en la zona de estudio.

Datos obtenidos a partir de la Base de Datos Climática de Marte (Forget el al., 1999; Lewis et al., 1999). 
Para entender mejor el comportamiento de este balance de flujo radiativo se han estudiado sus valores en las inmediaciones de la zona de estudio mediante dos gráficas, una por el flujo saliente y otra por el entrante (Figura 8, arriba). En ella se observa como solo existe flujo solar de onda corta durante el día, como era de esperar; siendo cero durante el la noche. Las horas de luz se encuentran entre las 2 y las 12 horas, hallando en ambos casos valores máximos entre las 6 y las 8 horas $\left(541.8 \mathrm{~W} / \mathrm{m}^{2}\right.$ en flujo entrante y $105,0 \mathrm{~W} / \mathrm{m}^{2}$ en flujo saliente). Hay una considerable variación anual, siendo máximo el flujo en el mes 7 para ambos flujos y mínimo en el mes 10 para el flujo entrante y en el mes 4 para el saliente. Los meses 1 y 4 tienen tendencias muy similares.

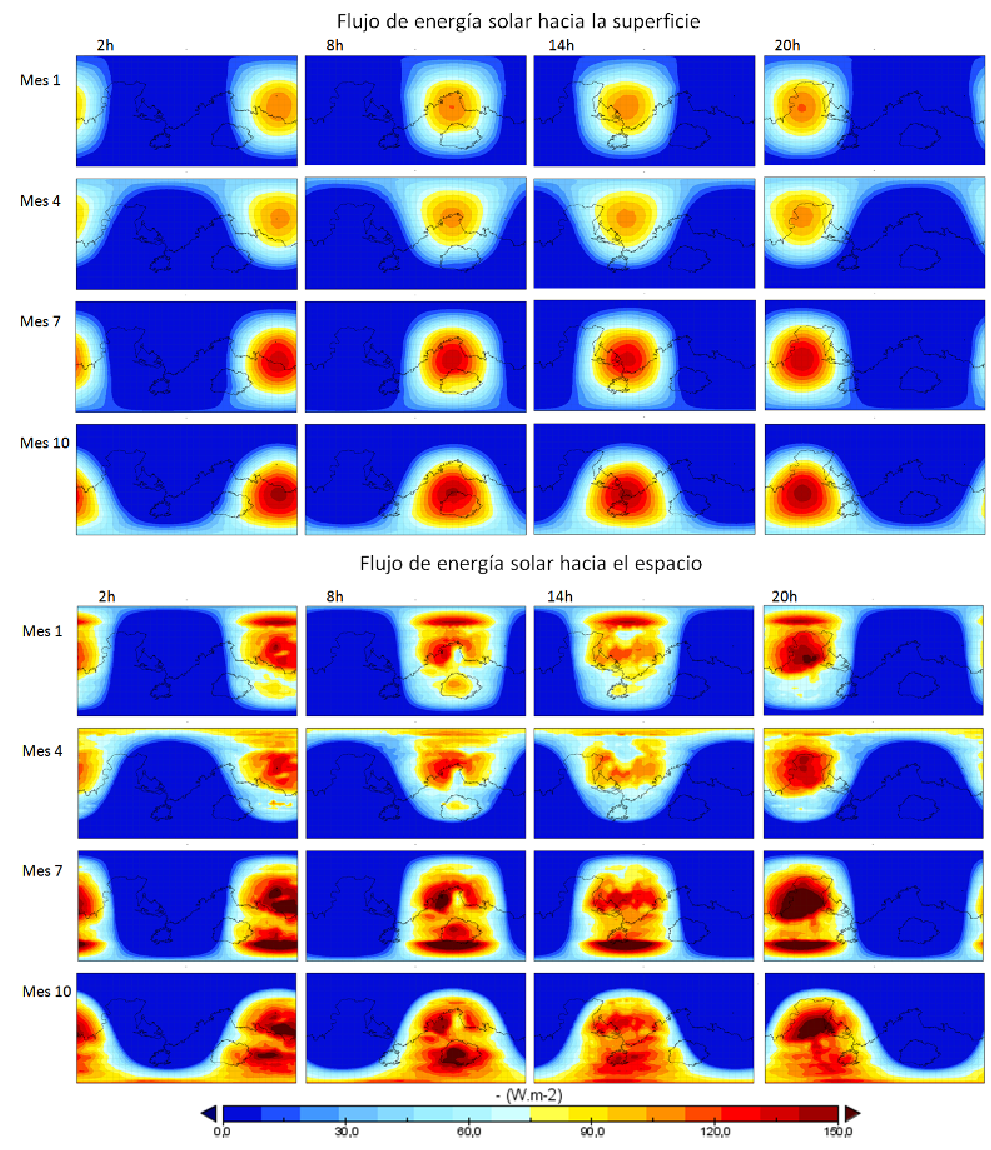

Figura 7. Mapas de flujos de energía solar hacia la superficie (entrante, arriba) y hacia el espacio (saliente, abajo). Las columnas representan diferentes horas $(2,8,14$ y 20) y las filas diferentes meses $(1,4,7$ y 10$)$. La línea negra representa el nivel de referencia: altitud 0m. Datos obtenidos a partir de la Base de Datos Climática de Marte (Forget el al., 1999; Lewis et al., 1999). 

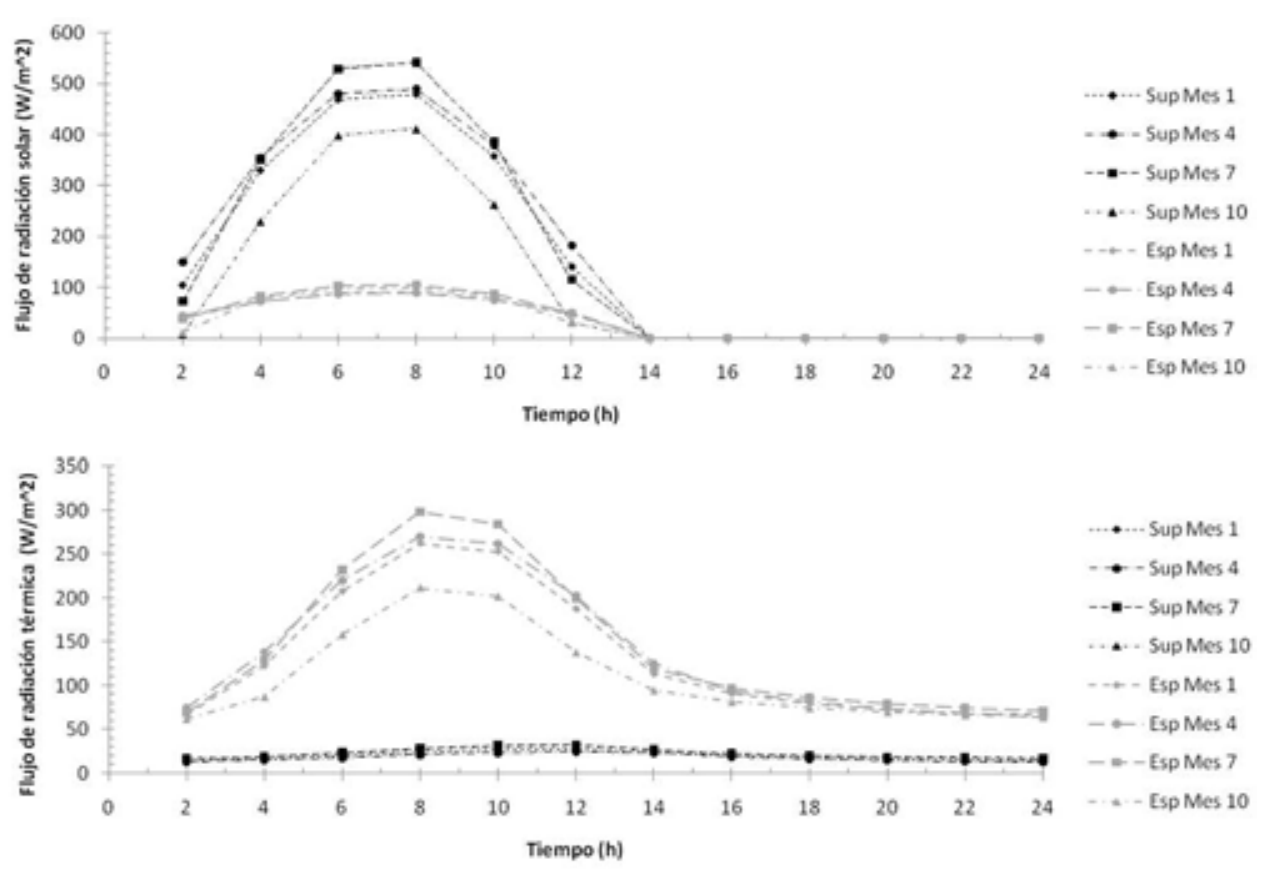

Figura 8. Gráficos de la variación diaria de los flujos de energía solar de onda corta (Arriba) y de los flujos de energía térmica de onda larga (IR, abajo) hacia la superficie (negro) y hacia el espacio (saliente, gris), para las inmediaciones de la zona de estudio.

Datos obtenidos a partir de la Base de Datos Climática de Marte (Forget el al., 1999; Lewis et al., 1999).

Se ha realizado el análisis anterior para el flujo de energía térmica de onda larga (IR), también tanto entrante como saliente. En la Figura 9 se muestran los mapas correspondientes.

En los mapas de flujo de energía térmica (IR) (Figura 9), tanto en el flujo saliente como entrante, se encuentran correlacionados con la incidencia de la radiación solar y el aumento de temperatura de la superficie del suelo. Esta relación está más clara en el caso de la radiación térmica que viaja hacia el espacio, siguiendo un esquema muy similar al que se ha observado a lo largo de este capítulo. Aunque se aprecia cierta influencia de la topografía, es en el caso del flujo hacia la superficie donde esta influencia es más clara. En ambos casos se aprecia como los valores de radiación térmica son considerablemente mayores durante los meses 7 y 10 (momento en que la energía se concentra en el hemisferio sur), que durante los meses 1 y 4 (cuando la radiación solar incide con más intensidad en el hemisferio norte). Los valores máximos para el flujo entrante se localizan dentro de las cuencas de Hellas y Argyre, mientras que para el flujo saliente se encuentran a latitudes más al norte (Figura 9). 


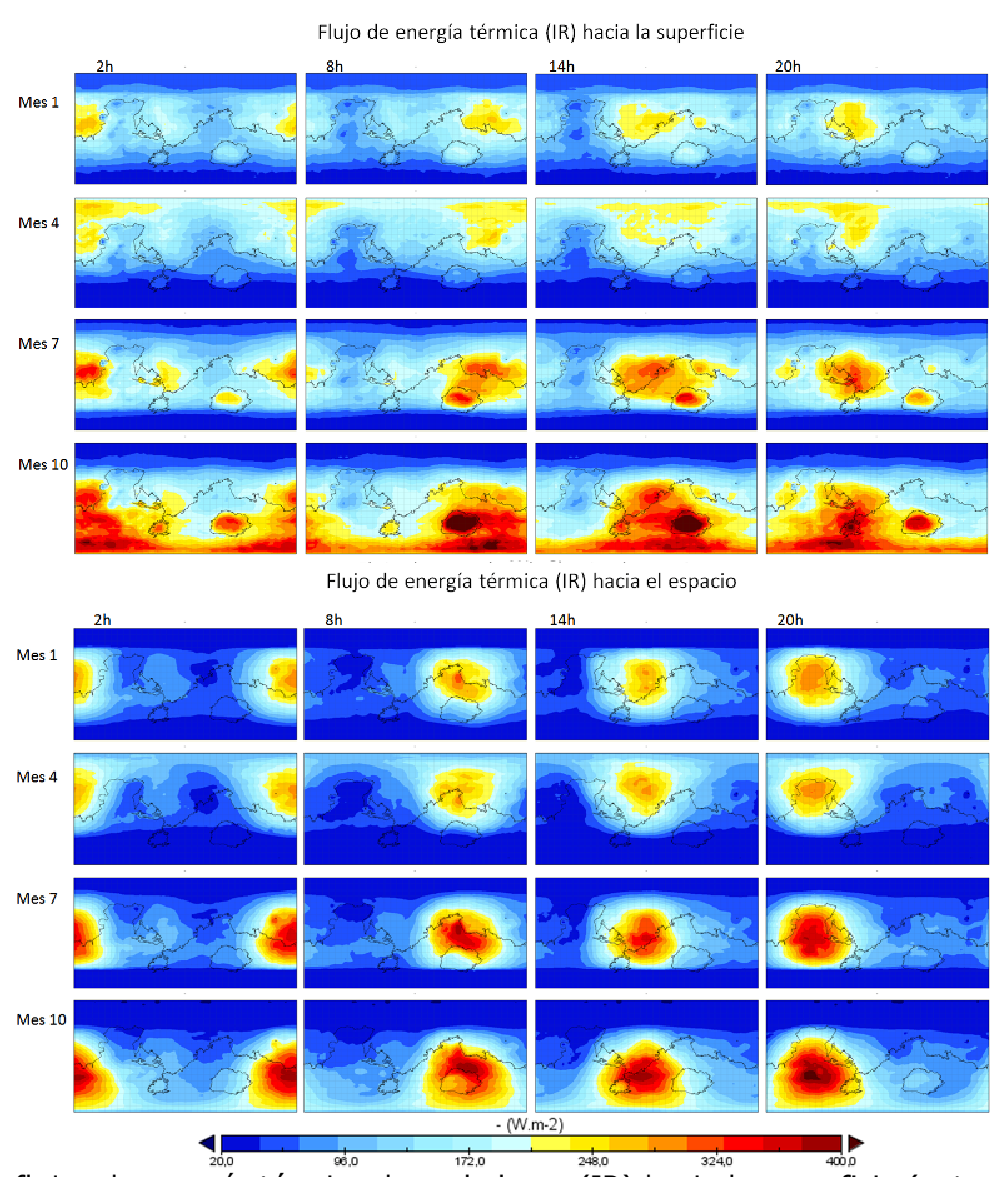

Figura 9. Mapas de flujos de energía térmica de onda larga (IR) hacia la superficie (entrante, arriba) y hacia el espacio (saliente, abajo). Las columnas representan los valores a diferentes horas $(2,8,14$ y 20$)$ y las filas diferentes meses $(1,4,7$ y 10). La línea negra representa el nivel de referencia: altitud Om sobre la superficie del suelo. Datos obtenidos a partir de la Base de Datos Climática de Marte (Forget el al., 1999; Lewis et al., 1999).

En los gráficos de la Figura 8 (abajo) se aprecian tendencias similares a las encontradas en los flujos de radiación solar (Figura 8, arriba). Valores máximos a las 10 horas (un poco después para la entrante, y un poco antes para la saliente) y muy bajos durante la noche. En el flujo hacia la superficie los valores medios son bajos $(20,6$ $\left.\mathrm{W} / \mathrm{m}^{2}\right)$, en comparación con el flujo hacia el espacio $\left(131,5 \mathrm{~W} / \mathrm{m}^{2}\right)$. Los valores mínimos de radiación entrante se encuentran en torno a los $15 \mathrm{~W} / \mathrm{m}^{2}$, y los máximos $30 \mathrm{~W} / \mathrm{m}^{2}$. Para la radiación saliente, el mínimo ronda los $65 \mathrm{~W} / \mathrm{m}^{2}$ y el máximo los $260 \mathrm{~W} / \mathrm{m}^{2}$.

\section{OTRAS VARIABLES}

Una variable de interés que se puede obtener a partir de la Base de Datos Climática (Forget el al., 1999; Lewis et al., 1999) es la cubierta de hielo de $\mathrm{CO}_{2}$. En la Figura 10 (arriba), se muestra un mapa para la cubierta en el mes 12, mes en que la cubierta se encuentra sobre el polo norte y su extensión alcanza latitudes más australes. Incluso en este momento la cubierta queda muy lejos de la zona de estudio.

Por último, se analiza el espesor óptico atmosférico (dust optical depth), que nos permite conocer mejor este escenario. La variación durante el día, al igual que en la cubierta de hielo, es mínima; pero sí se producen variaciones anuales. Por ello, en la Figura 10 (abajo), se muestran los mapas del espesor óptico atmosférico para los cuatro meses que se han venido estudiando en este trabajo. Se puede ver que el mes con 
menor espesor óptico es el 10, mientras que durante el 4 se encontrarían valores más altos por todo el planeta. Las zonas con mayor opacidad atmosférica (mayor espesor óptico, por tanto) son las cuencas de Hellas y Argyre, Valles Marinieris y las Tierras Bajas, coincidiendo con las zonas de mejor altitud.

\section{CONCLUSIONES}

La temperatura atmosférica, según la $M C D$, ha resultado ser unos $40 \mathrm{~K}$ menor que la superficial en la zona de estudio durante el día, mientras que tiene unos valores muy similares a ésta durante la noche. El patrón de variabilidad es similar, aunque con menor amplitud térmica día-noche, con valores que se reducen a unos $50 \mathrm{~K}$, casi la mitad que en la temperatura superficial del suelo. El perfil altimétrico de la temperatura atmosférica, presenta interesantes variaciones anuales que tendrían implicaciones en la dinámica atmosférica, por ejemplo a nivel de formación de nubes, y que sería interesante estudiar más en detalle. En cualquier caso, la estructura es similar a la descrita en la bibliografía. La temperatura disminuye en la parte inferior (gradiente negativo, hasta los $45 \mathrm{~km}$ ), pero en la parte media (entre los 45 y los $110 \mathrm{~km}$ ) desciende ligeramente en la mayoría de meses, exceptuando el mes 4, donde se produciría un ligero aumento a partir de los $60 \mathrm{~km}$ de altitud (gradiente positivo). No se aprecia el aumento de las temperaturas esperado en la parte superior de la atmósfera, al contrario, la temperatura parece descender en las capas altas. Puede deberse a un fallo en el modelo, o a que esta se produzca en cotas mayores.
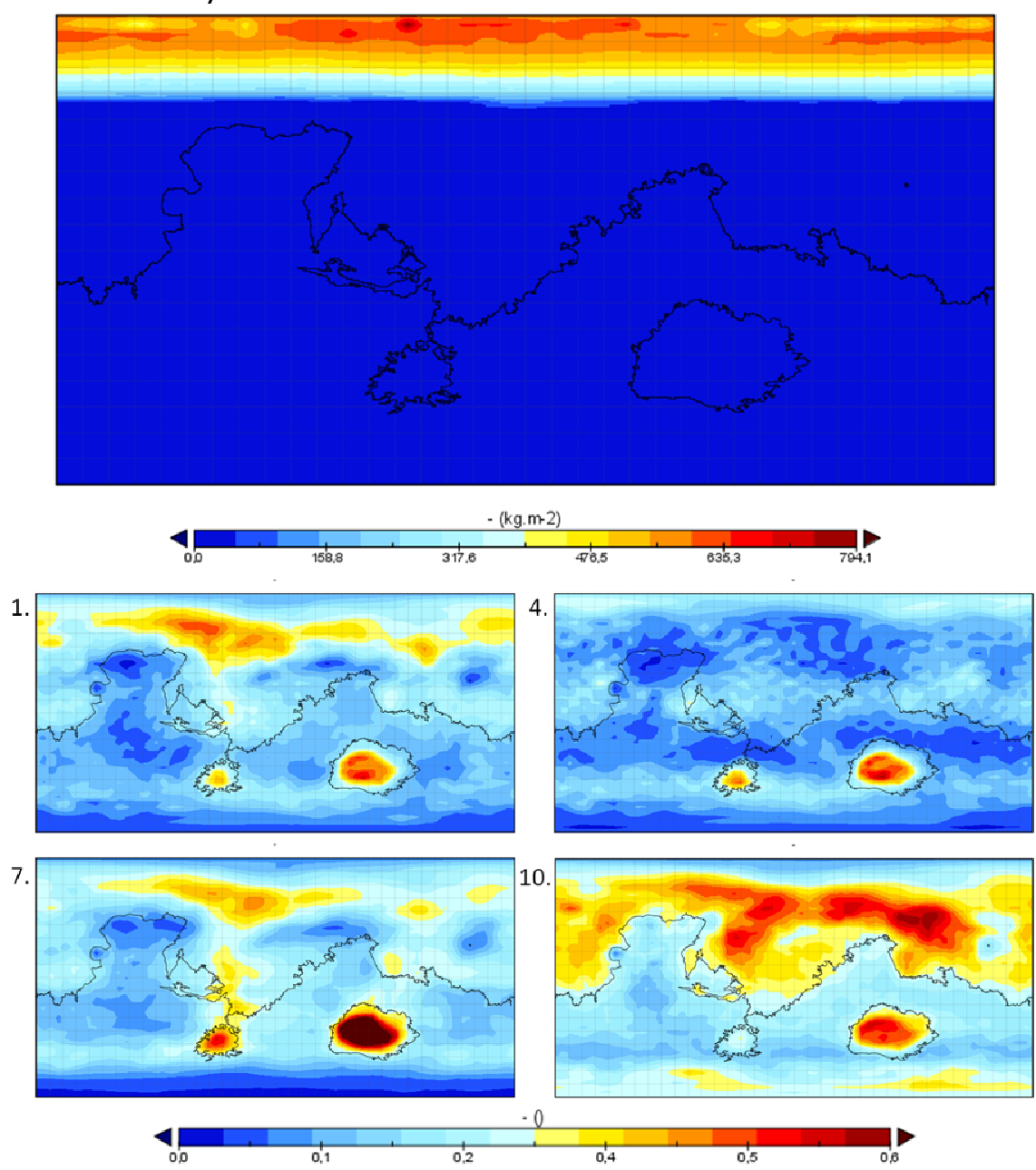

Figura 10. El mapa superior corresponde a la cubierta de hielo de $\mathrm{CO}_{2}$ en Marte, durante el mes 12 . Abajo, se representan mapas del espesor óptico (dust optical depth) en Marte, durante el los meses 1, 4, 7 y 10. La línea negra representa el nivel de referencia: altitud 0m sobre la superficie del suelo. Datos obtenidos a partir de la Base de Datos Climática de Marte (Forget el al., 1999; Lewis et al., 1999). 
En lo referente a las presiones superficiales, la presión media en la zona es de $562,9 \mathrm{~Pa}$ (Figura 6), un valor algo más bajo que la media planetaria (700 Pa; Barlow, 2008). Al contrario que como ocurría con las temperaturas, las mayores variabilidades de presión se producen a lo largo del año (con variaciones de hasta $80 \mathrm{~Pa}$ ), mientras que los valores son más o menos estables durante el día (con rangos de variación de $10 \mathrm{~Pa}$ ). Son especialmente interesantes los valores que superan los $611,73 \mathrm{~Pa}$, presión correspondiente al punto triple del agua (Haberle et al., 2001). Durante los meses 9, 10 y 11 la media superaría esta cifra, lo que podría tener importantes implicaciones en la presencia de agua líquida superficial y subsuperficial. Por último, los flujos de energía, que también se han analizado en este trabajo, pueden dar lugar a interesantes estudios sobre el balance de energía en la zona.

Podemos concluir que la Mars Climate Database es una herramienta sencilla y que aporta una gran cantidad de información con una más que aceptable resolución espacial y temporal; y que permite conocer como son las características y variabilidad del clima en una zona concreta del planeta Marte. Esto permite tener una idea previa de las condiciones que habrá de soportar una sonda que se envíe a este punto o de apoyo a la interpretación de los procesos físicos, químicos y biológicos que se produzcan sobre los materiales que componen la superficie y la atmosfera en la zona de amartizaje.

\section{BIBLIOGRAFÍA}

Anguita, F. (1998) Historia de Marte. Mito, exploración, futuro. Editorial Planeta (Barcelona) ISBN: 84-08-02698-4

Barlow, N. (2008) Mars, An introduction to its Interior, Surface and Atmosphere. Cambridge University Press (Nueva York) ISBN: 978-0-521-85226-5

Basu, S., Richardson, M.I., Y Wilson, R.J. (2004) Simulation of the martian dust cycle with the DFDL Mars GCM. Journal of Geophysical Research, 109. E11006

Clancy, R.T., Sandor, B.J., Wolff, M.J., Christensen, P.R., Smith, M.D., Pearl J.C., Conrath B.J., Wilson R.J. (2000) An intercomparison of ground-based millimeter, MGS TES, and Viking atmospheric temperature measurements: Seasonal and interannual variability of temperatures and dust loading in the global Mars atmosphere. Journal of Geophysical Research, Volume 105. 9553-9572.

De Pater, I., Lissauer, J.J. (2001) Planetary sciences. Cambridge Univerity Press (Cambridge) ISBN: 0-521-488219-4

Forget, F., Hourdin, F., Fournier, R., Hourdin, C., Talagrand, O., Collins, M., Lewis, S.R., Read, P.L. (1999) Improved general circulation models of the Martian atmosphere from the surface to above $80 \mathrm{~km}$. Journal of Geophysics Research, 104, 24.15524.176.

Forget, F., Costard, F., Lognonné, P. (2006) Planet Mars, Story of another world. Springer - Praxis (Berlín) ISBN: 978-0-0387-48925-4

Greeley, R., Whelley, P.L., Arvidson, R.E., Cabrol, N.A., Foley, D.F., Franklin, B.J., Geissler, P.G., Golombek, M.P., Kuzmin, R.O., Landis, G.A., Lemmon, M.T., Neakrase, L.D.V., Squyres, S.W., Thompson, S.D. (2006), Active dust devils in Gusev crater, Mars: Observations from the Mars Exploration Rover Spirit, J. Geophys. Res., 111, E12S09, doi:10.1029/2006JE002743

Haberle, R.M., McKay, C.P., Schaeffer, J., Cabrol, N.A., Grin, E. A., Zent, A.P. y Quinn, R. (2001) On the possibility of liquid water on present-day Mars. Journal of Geophysical Research, 23. 317-323, 326.

Hinson, D.P, Simpson, R.A, Twicken, J.D., Tyler, G.L., Flasar, F.M. (1999) Initial results for radio occultation measurements with Mars Global surveyor. Journal of Geophysical Research, 104. 26997-27012.

James, P.B., Kieffer, H.H., Paige, D.A. (1992) The seasonal cycle of carbon dioxide on Mars. En: Mars (A93-27852 09-91), p. 934-968. 
James, P.B, Hollingsworth, J.L., Wolff, M.J., Lee, S.W. (1999) North Polar Dust Storms in Early Spring on Mars. Icarus, 138 (1). 64-73. ISSN 0019-1035

Joshi, M.M., LeWIS, S.R., ReAD, P.L., CATLING, D.C. (1995) Western boundary currents in the martian atmosphere: numerical observations and observational evidence. Journal of Geophysical Research, 100. 5485-5500.

KAHRE, R.A, MuRPhy, J.R., HABerLe, R.M. (2006) Modeling the martian dust cycle and surface dust reservoirs with the NASA Ames general circulation model. Journal of Geophysical Research-Planets, 111. E060008.

Kieffer, H.H., Martin, T.Z, Peterfreund, A.R., Jakosky, B.M. (1977) Thermal and albedo mapping of Mars during the Viking primary mission. Journal of Geophysical Research, 82. 4249-4291.

LEOVY, C. (2001) Weather and climate on Mars. Nature. 245-249.

Lewis, S.R., Collins, M., ReAd, P.L., Forget, F., Hourdin, F., Fournier, R., Hourdin, C., TALAGRAND, O., HUOT, J.P. (1999) A climate database for Mars. Journsl of Geophysical Research-Planets, 104. 24,177-24,194.

Millour, E., Forget, F. (2008) Mars Climate Database Version 4.3, Validation document (Borrador).

SChofield, J.T., Barnes, J.R., Crisp, D., HABerle, R.M., LARSEn, S., Magalhães, J.A., MURPHY, J.R., SEIFF, A., WILSON, G. (1997) The Mars Pathfinder Atmospheric Structure Investigation/Meteorology (ASI/MET) experiment. Science, 278. 1752-1758.

Smith, P., Renno, N. (2001) Studying Earth dust devils for possible Mars mission. UniSci News. 